CRYSTALLOGRAPHIC COMMUNICATIONS

ISSN 2056-9890

Received 11 July 2015

Accepted 12 August 2015

Edited by M. Gdaniec, Adam Mickiewicz University, Poland

Keywords: crystal structure; nitrobenzenesulfonate; $\mathrm{N}$ (nitro) ...O interactions; $\mathrm{C}-\mathrm{H}$... O interactions; $\pi-\pi$ interactions

CCDC reference: 1418463

Supporting information: this article has supporting information at journals.iucr.org/e

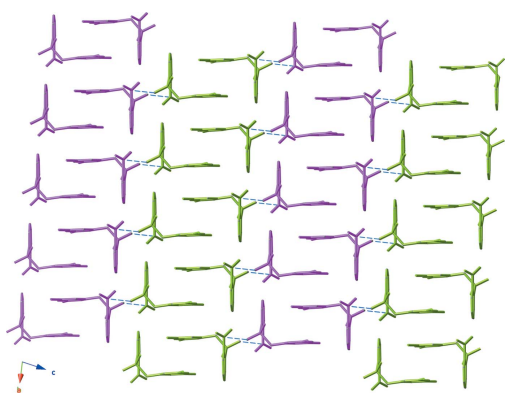

OPEN $\odot$ ACCESS

\section{Crystal structure of 3,5-dimethylphenyl 2-nitro- benzenesulfonate}

Tsvetelina P. Atanasova, ${ }^{a}$ Sean Riley, ${ }^{a}$ Shannon M. Biros, ${ }^{a}$ Richard J. Staples ${ }^{b}$ and Felix N. Ngassa ${ }^{a} *$

${ }^{\mathbf{a}}$ Department of Chemistry, Grand Valley State University, 1 Campus Dr., Allendale, MI 49401, USA, and ${ }^{\mathbf{b}}$ Center for Crystallographic Research, Department of Chemistry, Michigan State University, 578 S. Shaw Lane, East Lansing, MI 48824, USA. *Correspondence e-mail: ngassaf@gvsu.edu

The title compound, $\mathrm{C}_{14} \mathrm{H}_{13} \mathrm{NO}_{5} \mathrm{~S}$, was synthesized via a nucleophilic substitution reaction between 3,5-dimethylphenol and 2-nitrobenzenesulfonyl chloride. The aromatic rings attached to the $\mathrm{SO}_{3}$ group are oriented in a gauche fashion around the ester $\mathrm{S}-\mathrm{O}$ bond, with a $\mathrm{C}-\mathrm{S}-\mathrm{O}-\mathrm{C}$ torsion angle of $84.68(11)^{\circ}$. The molecules form centrosymmetric dimers via $\pi-\pi$ stacking interactions between 3,5-dimethylphenyl groups (centroid-centroid distance = 3.709 $\AA$ ). An intermolecular $\mathrm{S}=\mathrm{O} \cdots \mathrm{N}$ interaction between the sulfonyl and nitro groups, with an $\mathrm{O} \cdots \mathrm{N}$ distance of 2.9840 (18) $\AA$, organizes the dimers into columns extending along [011]. These columns are further assembled into (111) layers through $\mathrm{C}-\mathrm{H} \cdots \mathrm{O}$ interactions.

\section{Chemical context}

Microtubules form a major component of the cytoskeleton and have been implicated in a wide variety of cellular functions, such as cell division (Jordan \& Wilson, 2004). Microtubules therefore have been targeted in the design of drugs for the treatment of various forms of cancer (Spencer \& Faulds, 1994; Teicher, 2008; Trivedi et al., 2008). For example, Combretastatin A-4 (CA-4) has been shown to target tumor vasculature (Griggs et al., 2001). Most known antimicrotubules have poor biopharmaceutical properties, uch as chemoresistance and toxicity (Islam et al., 2003; Fortin et al., 2011).

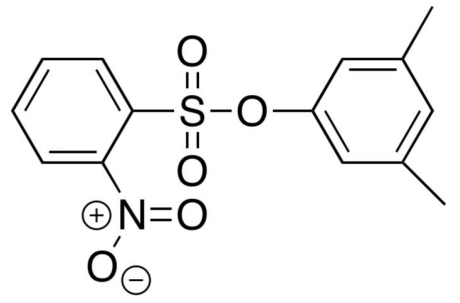

Research in the field for the synthesis of new antimicrotubule compounds has been geared towards compounds with improved biopharmaceutical properties (Fortin et al., 2011). To this end, Fortin and co-workers have designed and synthesized various sulfonate derivatives, which have been

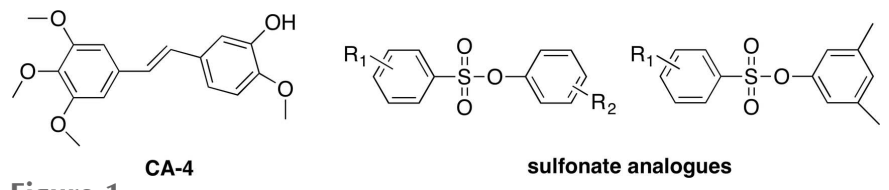

Figure 1

The structures of CA- 4 and sulfonate analogues, where $R_{1}$ and $R_{2}$ are substituents on the sulfonyl and phenoxy benzene rings, respectively. 


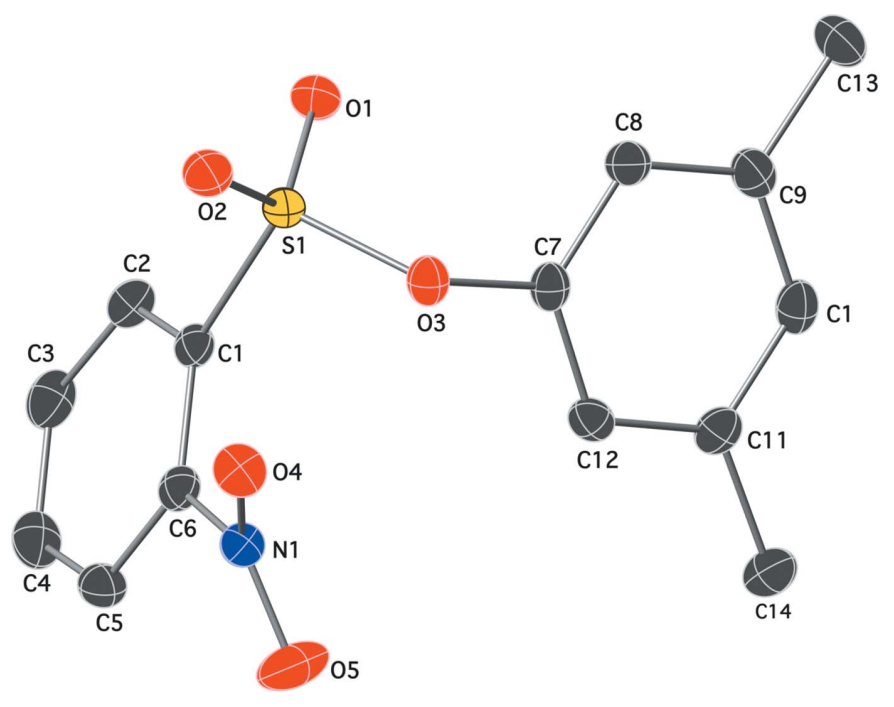

Figure 2

The molecular structure of the title compound, with displacement ellipsoids shown at the $50 \%$ probability level. All $\mathrm{H}$ atoms have been omitted for clarity. Color codes: black C, blue N, red O and yellow S.

tested as new tubulin inhibitors mimicking Combretastatin A-4 (Fig. 1).

A series of sulfonate derivatives have shown promise as anticancer drugs, with some having lower toxicity than CA-4 (Fortin et al., 2011). We embarked on the synthesis of sulfonate derivatives with the long-term goal of investigating the effect of the benzene-ring substituents on the cytotoxicity of the sulfonate derivatives. To the best of our knowledge, despite the simplicity of the sulfonate derivatives, there has been no relevant previous crystallographic studies. Therefore, we report here the synthesis and crystal structure of 3,5-dimethylphenyl 2-nitrobenzenesulfonate.

\section{Structural commentary}

In the title molecule (Fig. 2), the $\mathrm{O} 1=\mathrm{S} 1=\mathrm{O} 2$ and $\mathrm{C} 1-\mathrm{S} 1-$ O3 bond angles of 119.41 (7) and $104.16(6)^{\circ}$ are typical for phenyl benzenesulfonates with a gauche conformation around the ester $\mathrm{S}-\mathrm{O}$ bond. The torsion angle $\mathrm{C} 1-\mathrm{S} 1-\mathrm{O} 3-\mathrm{C} 7$ around the ester bond is $-84.68(11)^{\circ}$. Owing to steric hindrance between the ortho substituents of the benzene ring, the nitro group is twisted relative to the benzene best plane by

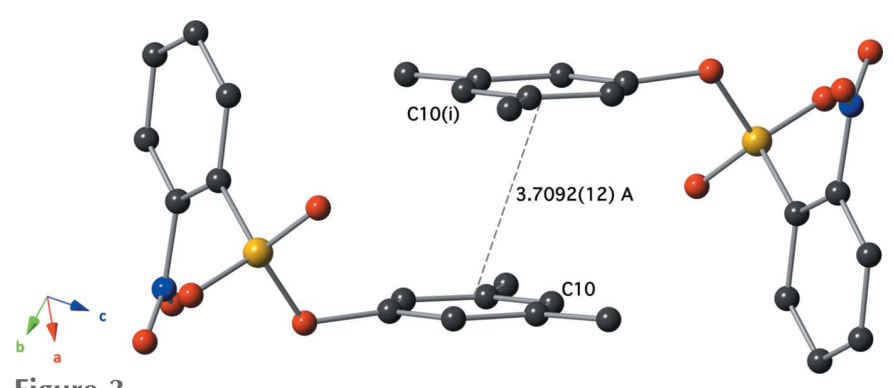

Figure 3

The centrosymmetric dimers formed by intermolecular offset $\pi-\pi$ stacking interactions. [Symmetry code: (i) $-x+1,-y+2,-z$.]
Table 1

Hydrogen-bond geometry $\left(\AA{ }^{\circ}\right)$.

\begin{tabular}{lllll}
\hline$D-\mathrm{H} \cdots A$ & $D-\mathrm{H}$ & $\mathrm{H} \cdots A$ & $D \cdots A$ & $D-\mathrm{H} \cdots A$ \\
\hline $\mathrm{C} 5-\mathrm{H} 5 \cdots \mathrm{O} 1^{\mathrm{i}}$ & 0.95 & 2.56 & $3.4544(19)$ & 156 \\
$\mathrm{C} 8-\mathrm{H} 8 \cdots 5^{\mathrm{ii}}$ & 0.95 & 2.56 & $3.468(2)$ & 160 \\
\hline
\end{tabular}

Symmetry codes: (i) $x+1, y-1, z$; (ii) $x-1, y+1, z$.

$39.91(2)^{\circ}$, so that the shortest contact of 2.7941 (16) $\AA$ between the $\mathrm{O}$ atoms of these groups is close to the sum of the van der Waals radii.

\section{Supramolecular features}

The molecules of the title compound form centrosymmetric dimers via intermolecular $\pi-\pi$ stacking interactions between the relatively electron-rich $\mathrm{C} 7-\mathrm{C} 12$ benzene rings (Fig. 3), with a plane-to-plane distance of 3.4147 (15) $\AA$. The aromatic rings are stacked with an offset, and the distance between the centroids of these rings is 3.709 (12) $\AA$. Another centrosymmetric dimer is formed by an $\mathrm{S}=\mathrm{O} \cdots \mathrm{N}$ interaction, with an $\mathrm{N} 1 \cdots \mathrm{O} 2$ interatomic distance of 2.9840 (18) $\AA$. O $\cdots \mathrm{N}$ (nitro) interactions between nitro groups have been discussed in the literature (Daszkiewicz, 2013; Caracelli et al., 2014) and we report here the case of sulfonyl and nitro group interactions. Both types of dimers are assembled into a column-type structure extending along [011] (Fig. 4).

There are no classical hydrogen bonds in the crystal structure; however, nonclassical $\mathrm{C}-\mathrm{H} \cdots \mathrm{O}$ interactions between aromatic-ring $\mathrm{H}$ atoms and sulfonyl and nitro group $\mathrm{O}$ atoms organize the [011] columns into (111) layers. The geometry of these interactions is given in Table 1 .

\section{Database survey}

The Cambridge Structural Database (CSD, Version 5.36 with two updates; Groom \& Allen, 2014) contains three structures with an $o$-nitroarylsulfonyl moiety bonded to an aromatic ring through an ester linkage. These are CSD refcodes FEMQUK (Ichikawa et al., 2004), MIBZUT (Pelly et al., 2007), and FEMRIZ (Ichikawa et al., 2004). Like in the title compound, the aromatic substituents of the $\mathrm{SO}_{3}$ group are oriented gauche around the ester $\mathrm{S}-\mathrm{O}$ bond and the absolute value of the $\mathrm{C}-\mathrm{S}-\mathrm{O}-\mathrm{C}$ torsion angle is in the range 85.9 (3)$103.43(13)^{\circ}$. In each of these structures there are either intraor intermolecular $\mathrm{S}=\mathrm{O} \cdots \mathrm{N}$ interactions between the sulfonate and $o$-nitro groups.

\section{Synthesis and crystallization}

3,5-Dimethylphenol (2.44 g, $20 \mathrm{mmol})$ was dissolved in chilled dichloromethane $(25 \mathrm{ml})$. This was followed by the addition of pyridine $(3.2 \mathrm{ml}, 40 \mathrm{mmol})$. The resulting solution was cooled in an ice bath under an $\mathrm{N}_{2}$ atmosphere, followed by the addition of 2-nitrobenzenesulfonyl chloride (4.43 g, $20 \mathrm{mmol}$ ) portion-wise. The mixture was stirred at $273 \mathrm{~K}$ for $30 \mathrm{mins}$ and then at room temperature for $24 \mathrm{~h}$. The product precipitated 


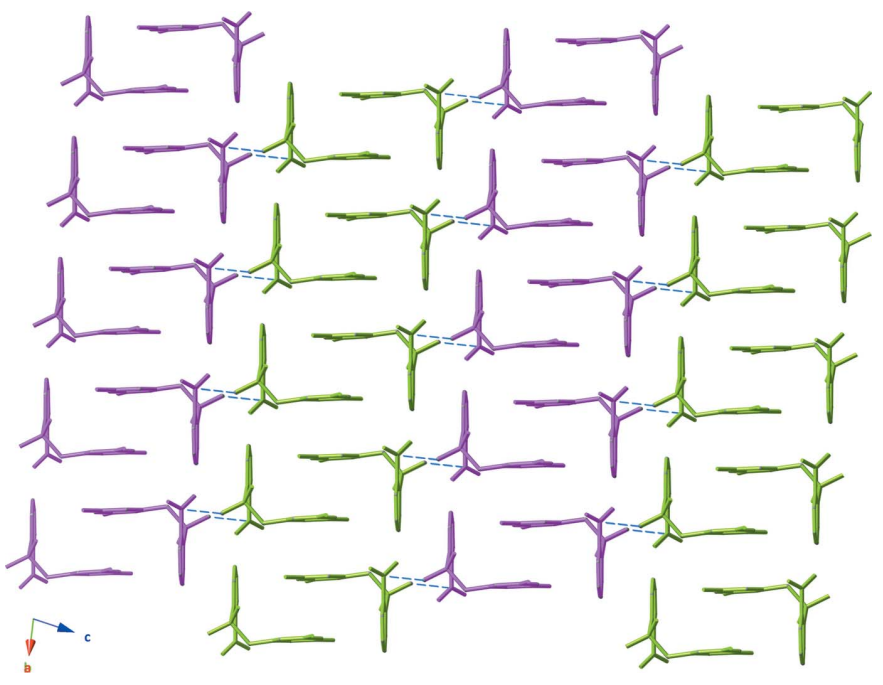

Figure 4

The packing of molecules in the crystal, viewed down the [110] direction. Columns of dimers formed via stacking interactions are colored green and pink in an alternating fashion, and potential $\mathrm{N} \cdots \mathrm{O}=\mathrm{S}$ interactions are denoted with blue dashed lines.

from the reaction mixture after sitting at $277 \mathrm{~K}$ for two weeks. The product was redissolved in dichloromethane, and the solvent was allowed to evaporate slowly to give large blockshaped crystals that were suitable for analysis by X-ray diffraction (m.p. 374-378 K).

\section{Refinement}

Crystal data, data collection and structure refinement details are summarized in Table 2. $\mathrm{H}$ atoms were placed in calculated positions and constrained to ride on their parent atoms, with $U_{\text {iso }}(\mathrm{H})=1.2 U_{\text {eq }}(\mathrm{C})$ for $\mathrm{CH}$ groups and $1.5 U_{\text {eq }}(\mathrm{C})$ for methyl groups.

\section{Acknowledgements}

The authors thank GVSU for financial support (Weldon Fund, CSCE), the NSF for a $300 \mathrm{MHz}$ Jeol FT-NMR (CCLI0087655), and Pfizer, Inc. for the donation of a Varian Inova 400 FT-NMR. The CCD-based X-ray diffractometers at Michigan State University were upgraded and/or replaced by departmental funds.

\section{References}

Bourhis, L. J., Dolomanov, O. V., Gildea, R. J., Howard, J. A. K. \& Puschmann, H. (2015). Acta Cryst. A71, 59-75.

Bruker (2013). APEX2, SAINT and SADABS. Bruker AXS Inc., Madison, Wisconsin, USA.
Table 2

Experimental details.

\begin{tabular}{ll}
\hline Crystal data & \\
Chemical formula & $\mathrm{C}_{14} \mathrm{H}_{13} \mathrm{NO}_{5} \mathrm{~S}$ \\
$M_{\mathrm{r}}$ & 307.31 \\
Crystal system, space group & Triclinic, $P \overline{1}$ \\
Temperature $(\mathrm{K})$ & 173 \\
$a, b, c(\AA)$ & $7.9958(4), 7.9991(5), 12.0238(3)$ \\
$\alpha, \beta, \gamma\left({ }^{\circ}\right)$ & $83.908(3), 76.286(3), 63.411(4)$ \\
$V\left(\AA^{3}\right)$ & $668.10(6)$ \\
$Z$ & 2 \\
Radiation type & $\mathrm{Cu} K \alpha$ \\
$\mu\left(\mathrm{mm}^{-1}\right)$ & 2.37 \\
Crystal size $(\mathrm{mm})$ & $0.38 \times 0.34 \times 0.21$ \\
& \\
Data collection & $\mathrm{Bruker}$ APEXII CCD \\
Diffractometer & Multi-scan $(S A D A B S ;$ Bruker, \\
Absorption correction & $2013)$ \\
& $0.630,0.754$ \\
$T_{\min }, T_{\max }$ & $10317,2519,2461$ \\
No. of measured, independent and & \\
$\quad$ observed $[I>2 \sigma(I)]$ reflections & 0.022 \\
$R_{\text {int }}$ & 0.617 \\
(sin $\theta / \lambda)_{\text {max }}\left(\AA^{-1}\right)$ & \\
Refinement & \\
$R\left[F^{2}>2 \sigma\left(F^{2}\right)\right], w R\left(F^{2}\right), S$ & $0.035,0.092,1.05$ \\
No. of reflections & 2519 \\
No. of parameters & 192 \\
$\mathrm{H}-$-atom treatment & $\mathrm{H}$-atom parameters constrained \\
$\Delta \rho_{\text {max }}, \Delta \rho_{\text {min }}\left(\mathrm{e} \AA^{-3}\right)$ & $0.33,-0.50$ \\
\hline
\end{tabular}

Computer programs: APEX2 and SAINT (Bruker, 2013), SHELXS97 (Sheldrick, 2008), SHELXL2014 (Sheldrick, 2015), CrystalMaker (Palmer, 2007) and OLEX2 (Dolomanov et al., 2009; Bourhis et al., 2015).

Caracelli, I., Maganhi, S. H., Moran, P. J. S., Paula, B. R. S. de, Delling, F. N. \& Tiekink, E. R. T. (2014). Acta Cryst. E70, o1051-o1052.

Daszkiewicz, M. (2013). CrystEngComm, 15, 10427-10430.

Dolomanov, O. V., Bourhis, L. J., Gildea, R. J., Howard, J. A. K. \& Puschmann, H. (2009). J. Appl. Cryst. 42, 339-341.

Fortin, S., Wei, L., Moreau, E., Lacroix, J., Côté, M.-F., Petitclerc, É., Kotra, L. P. \& Gaudreault, R. C. (2011). J. Med. Chem. 54, 45594580.

Griggs, J., Metcalfe, J. C. \& Hesketh, R. (2001). Lancet Oncol. 2, 8287.

Groom, C. R. \& Allen, F. H. (2014). Angew. Chem. Int. Ed. 53, 662671.

Ichikawa, M., Takahashi, M., Aoyagi, S. \& Kibayashi, C. (2004). J. Am. Chem. Soc. 126, 16553-16558.

Islam, M. N., Song, Y. \& Iskander, M. N. (2003). J. Mol. Graph. Model. 21, 263-272.

Jordan, M. A. \& Wilson, L. (2004). Nat. Rev. Cancer, 4, 253-265.

Palmer, D. (2007). CrystalMaker. CrystalMaker Software, Bicester, England.

Pelly, S. C., Govener, S., Fernades, M. A., Schmalz, H.-G. \& de Koning, C. B. (2007). J. Org. Chem. 72, 2857-2864.

Sheldrick, G. M. (2008). Acta Cryst. A64, 112-122.

Sheldrick, G. M. (2015). Acta Cryst. C71, 3-8.

Spencer, C. M. \& Faulds, D. (1994). Drugs, 48, 794-847.

Teicher, B. A. (2008). Clin. Cancer Res. 14, 1610-1617.

Trivedi, M., Budihardjo, I., Loureiro, K., Reid, T. R. \& Ma, J. D. (2008). Future Oncol. 4, 483-500. 


\section{supporting information}

Acta Cryst. (2015). E71, 1045-1047 [https://doi.org/10.1107/S2056989015015078]

\section{Crystal structure of 3,5-dimethylphenyl 2-nitrobenzenesulfonate}

Tsvetelina P. Atanasova, Sean Riley, Shannon M. Biros, Richard J. Staples and Felix N. Ngassa

Computing details

Data collection: APEX2 (Bruker, 2013); cell refinement: SAINT (Bruker, 2013); data reduction: SAINT (Bruker, 2013); program(s) used to solve structure: SHELXS97 (Sheldrick, 2008); program(s) used to refine structure: SHELXL2014

(Sheldrick, 2015); molecular graphics: CrystalMaker (Palmer, 2007); software used to prepare material for publication: OLEX2 (Dolomanov et al., 2009; Bourhis et al., 2015).

3,5-Dimethylphenyl 2-nitrobenzenesulfonate

Crystal data

$\mathrm{C}_{14} \mathrm{H}_{13} \mathrm{NO}_{5} \mathrm{~S}$

$M_{r}=307.31$

Triclinic, $P \overline{1}$

$a=7.9958(4) \AA$

$b=7.9991(5) \AA$

$c=12.0238(3) \AA$

$\alpha=83.908(3)^{\circ}$

$\beta=76.286(3)^{\circ}$

$\gamma=63.411(4)^{\circ}$

$V=668.10(6) \AA^{3}$

\section{Data collection}

Bruker APEXII CCD

diffractometer

Radiation source: sealed tube

Graphite monochromator

Detector resolution: 8 pixels $\mathrm{mm}^{-1}$

$\varphi$ and $\omega$ scans

Absorption correction: multi-scan

(SADABS; Bruker, 2013)

$T_{\min }=0.630, T_{\max }=0.754$

Refinement

Refinement on $F^{2}$

Least-squares matrix: full

$R\left[F^{2}>2 \sigma\left(F^{2}\right)\right]=0.035$

$w R\left(F^{2}\right)=0.092$

$S=1.05$

2519 reflections

192 parameters

0 restraints
$Z=2$

$F(000)=320$

$D_{\mathrm{x}}=1.528 \mathrm{Mg} \mathrm{m}^{-3}$

$\mathrm{Cu} K \alpha$ radiation, $\lambda=1.54178 \AA$

Cell parameters from 8863 reflections

$\theta=3.8-72.3^{\circ}$

$\mu=2.37 \mathrm{~mm}^{-1}$

$T=173 \mathrm{~K}$

Block, colourless

$0.38 \times 0.34 \times 0.21 \mathrm{~mm}$

10317 measured reflections

2519 independent reflections

2461 reflections with $I>2 \sigma(I)$

$R_{\text {int }}=0.022$

$\theta_{\text {max }}=72.1^{\circ}, \theta_{\min }=3.8^{\circ}$

$h=-9 \rightarrow 9$

$k=-9 \rightarrow 9$

$l=-14 \rightarrow 14$

Primary atom site location: structure-invariant direct methods

Hydrogen site location: inferred from neighbouring sites

$\mathrm{H}$-atom parameters constrained

$w=1 /\left[\sigma^{2}\left(F_{\mathrm{o}}^{2}\right)+(0.0559 P)^{2}+0.3536 P\right]$

where $P=\left(F_{\mathrm{o}}{ }^{2}+2 F_{\mathrm{c}}{ }^{2}\right) / 3$

$(\Delta / \sigma)_{\max }<0.001$

$\Delta \rho_{\max }=0.33$ e $\AA^{-3}$

$\Delta \rho_{\min }=-0.50$ e $\AA^{-3}$ 


\section{Special details}

Geometry. All e.s.d.'s (except the e.s.d. in the dihedral angle between two 1.s. planes) are estimated using the full covariance matrix. The cell e.s.d.'s are taken into account individually in the estimation of e.s.d.'s in distances, angles and torsion angles; correlations between e.s.d.'s in cell parameters are only used when they are defined by crystal symmetry. An approximate (isotropic) treatment of cell e.s.d.'s is used for estimating e.s.d.'s involving l.s. planes.

Fractional atomic coordinates and isotropic or equivalent isotropic displacement parameters $\left(\AA^{2}\right)$

\begin{tabular}{|c|c|c|c|c|}
\hline & $x$ & $y$ & $z$ & $U_{\text {iso }} * / U_{\text {eq }}$ \\
\hline S1 & $0.32156(5)$ & $0.85859(5)$ & $0.36621(3)$ & $0.01694(13)$ \\
\hline $\mathrm{O} 1$ & $0.27175(16)$ & $1.05073(15)$ & $0.33973(10)$ & $0.0239(3)$ \\
\hline $\mathrm{O} 2$ & $0.22079(15)$ & $0.81004(16)$ & $0.46826(9)$ & $0.0240(3)$ \\
\hline $\mathrm{O} 3$ & $0.29830(15)$ & $0.76150(15)$ & $0.26599(9)$ & $0.0193(2)$ \\
\hline $\mathrm{O} 4$ & $0.46725(16)$ & $0.43665(16)$ & $0.39045(10)$ & $0.0240(3)$ \\
\hline O5 & $0.73130(19)$ & $0.28883(18)$ & $0.26840(12)$ & $0.0375(3)$ \\
\hline N1 & $0.62367(18)$ & $0.41785(18)$ & $0.33520(11)$ & $0.0201(3)$ \\
\hline $\mathrm{C} 1$ & $0.5719(2)$ & $0.7475(2)$ & $0.36177(12)$ & $0.0171(3)$ \\
\hline $\mathrm{C} 2$ & $0.6508(2)$ & $0.8662(2)$ & $0.37453(13)$ & $0.0216(3)$ \\
\hline $\mathrm{H} 2$ & 0.5728 & 0.9973 & 0.3801 & $0.026^{*}$ \\
\hline $\mathrm{C} 3$ & $0.8425(2)$ & $0.7951(3)$ & $0.37920(14)$ & $0.0258(4)$ \\
\hline H3 & 0.8939 & 0.8771 & 0.3902 & $0.031 *$ \\
\hline $\mathrm{C} 4$ & $0.9587(2)$ & $0.6047(3)$ & $0.36792(14)$ & $0.0265(4)$ \\
\hline $\mathrm{H} 4$ & 1.0898 & 0.5564 & 0.3711 & $0.032 *$ \\
\hline $\mathrm{C} 5$ & $0.8841(2)$ & $0.4845(2)$ & $0.35195(13)$ & $0.0227(3)$ \\
\hline H5 & 0.9643 & 0.3542 & 0.3426 & $0.027^{*}$ \\
\hline C6 & $0.6920(2)$ & $0.5556(2)$ & $0.34977(12)$ & $0.0182(3)$ \\
\hline $\mathrm{C} 7$ & $0.3244(2)$ & $0.8273(2)$ & $0.15112(12)$ & $0.0175(3)$ \\
\hline $\mathrm{C} 8$ & $0.1844(2)$ & $0.9933(2)$ & $0.12119(13)$ & $0.0200(3)$ \\
\hline H8 & 0.0770 & 1.0683 & 0.1773 & $0.024 *$ \\
\hline $\mathrm{C} 9$ & $0.2044(2)$ & $1.0482(2)$ & $0.00677(14)$ & $0.0214(3)$ \\
\hline $\mathrm{C} 10$ & $0.3619(2)$ & $0.9314(2)$ & $-0.07306(13)$ & $0.0217(3)$ \\
\hline H10 & 0.3744 & 0.9677 & -0.1513 & $0.026^{*}$ \\
\hline C11 & $0.5014(2)$ & $0.7635(2)$ & $-0.04207(13)$ & $0.0198(3)$ \\
\hline $\mathrm{C} 12$ & $0.4822(2)$ & $0.7111(2)$ & $0.07297(13)$ & $0.0186(3)$ \\
\hline H12 & 0.5755 & 0.5979 & 0.0971 & $0.022 *$ \\
\hline $\mathrm{C} 13$ & $0.0582(3)$ & $1.2305(2)$ & $-0.02968(16)$ & 0.0294 (4) \\
\hline $\mathrm{H} 13 \mathrm{~A}$ & -0.0609 & 1.2720 & 0.0288 & $0.044^{*}$ \\
\hline H13B & 0.0333 & 1.2131 & -0.1026 & $0.044^{*}$ \\
\hline $\mathrm{H} 13 \mathrm{C}$ & 0.1069 & 1.3249 & -0.0390 & $0.044^{*}$ \\
\hline $\mathrm{C} 14$ & $0.6690(2)$ & $0.6430(2)$ & $-0.13189(14)$ & $0.0249(3)$ \\
\hline $\mathrm{H} 14 \mathrm{~A}$ & 0.6251 & 0.6435 & -0.2015 & $0.037^{*}$ \\
\hline H14B & 0.7263 & 0.5147 & -0.1032 & $0.037^{*}$ \\
\hline $\mathrm{H} 14 \mathrm{C}$ & 0.7647 & 0.6923 & -0.1494 & $0.037^{*}$ \\
\hline
\end{tabular}

Atomic displacement parameters $\left(\AA^{2}\right)$

\begin{tabular}{lllllll}
\hline & $U^{11}$ & $U^{22}$ & $U^{33}$ & $U^{12}$ & $U^{13}$ & $U^{23}$ \\
\hline S1 & $0.01488(19)$ & $0.0172(2)$ & $0.0159(2)$ & $-0.00464(15)$ & $-0.00261(13)$ & $-0.00112(14)$
\end{tabular}




\begin{tabular}{lllllll} 
O1 & $0.0254(6)$ & $0.0177(6)$ & $0.0258(6)$ & $-0.0055(4)$ & $-0.0081(5)$ & $-0.0009(5)$ \\
O2 & $0.0186(5)$ & $0.0292(6)$ & $0.0188(5)$ & $-0.0075(5)$ & $-0.0002(4)$ & $0.0000(5)$ \\
O3 & $0.0225(5)$ & $0.0206(5)$ & $0.0174(5)$ & $-0.0113(4)$ & $-0.0061(4)$ & $0.0026(4)$ \\
O4 & $0.0227(6)$ & $0.0257(6)$ & $0.0254(6)$ & $-0.0129(5)$ & $-0.0043(5)$ & $0.0023(5)$ \\
O5 & $0.0340(7)$ & $0.0311(7)$ & $0.0430(8)$ & $-0.0111(6)$ & $0.0021(6)$ & $-0.0198(6)$ \\
N1 & $0.0215(6)$ & $0.0181(6)$ & $0.0186(6)$ & $-0.0063(5)$ & $-0.0052(5)$ & $0.0000(5)$ \\
C1 & $0.0157(7)$ & $0.0211(7)$ & $0.0126(6)$ & $-0.0072(6)$ & $-0.0014(5)$ & $-0.0007(6)$ \\
C2 & $0.0236(7)$ & $0.0231(8)$ & $0.0179(7)$ & $-0.0108(6)$ & $-0.0017(6)$ & $-0.0027(6)$ \\
C3 & $0.0258(8)$ & $0.0362(9)$ & $0.0219(8)$ & $-0.0194(7)$ & $-0.0024(6)$ & $-0.0044(7)$ \\
C4 & $0.0170(7)$ & $0.0397(10)$ & $0.0227(8)$ & $-0.0124(7)$ & $-0.0034(6)$ & $-0.0006(7)$ \\
C5 & $0.0183(7)$ & $0.0240(8)$ & $0.0195(7)$ & $-0.0046(6)$ & $-0.0021(6)$ & $-0.0001(6)$ \\
C6 & $0.0187(7)$ & $0.0212(7)$ & $0.0137(7)$ & $-0.0087(6)$ & $-0.0017(5)$ & $-0.0006(6)$ \\
C7 & $0.0210(7)$ & $0.0189(7)$ & $0.0169(7)$ & $-0.0116(6)$ & $-0.0064(6)$ & $0.0016(6)$ \\
C8 & $0.0191(7)$ & $0.0189(7)$ & $0.0226(8)$ & $-0.0074(6)$ & $-0.0060(6)$ & $-0.0029(6)$ \\
C9 & $0.0244(7)$ & $0.0177(7)$ & $0.0256(8)$ & $-0.0098(6)$ & $-0.0114(6)$ & $0.0021(6)$ \\
C10 & $0.0304(8)$ & $0.0213(8)$ & $0.0181(7)$ & $-0.0145(7)$ & $-0.0086(6)$ & $0.0030(6)$ \\
C11 & $0.0238(7)$ & $0.0188(7)$ & $0.0208(7)$ & $-0.0127(6)$ & $-0.0038(6)$ & $-0.0023(6)$ \\
C12 & $0.0196(7)$ & $0.0146(7)$ & $0.0233(8)$ & $-0.0077(6)$ & $-0.0073(6)$ & $0.0010(6)$ \\
C13 & $0.0324(9)$ & $0.0217(8)$ & $0.0327(9)$ & $-0.0074(7)$ & $-0.0158(7)$ & $0.0046(7)$ \\
C14 & $0.0288(8)$ & $0.0225(8)$ & $0.0230(8)$ & $-0.0121(7)$ & $-0.0017(6)$ & $-0.0035(6)$ \\
& & & & & & \\
\hline
\end{tabular}

Geometric parameters $\left(\AA,{ }^{o}\right)$

\begin{tabular}{llll}
\hline $\mathrm{S} 1-\mathrm{O} 1$ & $1.4249(12)$ & $\mathrm{C} 7-\mathrm{C} 8$ & $1.380(2)$ \\
$\mathrm{S} 1-\mathrm{O} 2$ & $1.4198(11)$ & $\mathrm{C} 7-\mathrm{C} 12$ & $1.383(2)$ \\
$\mathrm{S} 1-\mathrm{O} 3$ & $1.5887(11)$ & $\mathrm{C} 8-\mathrm{H} 8$ & 0.9500 \\
$\mathrm{~S} 1-\mathrm{C} 1$ & $1.7797(15)$ & $\mathrm{C} 8-\mathrm{C} 9$ & $1.393(2)$ \\
$\mathrm{O} 3-\mathrm{C} 7$ & $1.4268(18)$ & $\mathrm{C} 9-\mathrm{C} 10$ & $1.394(2)$ \\
$\mathrm{O} 4-\mathrm{N} 1$ & $1.2195(17)$ & $\mathrm{C} 9-\mathrm{C} 13$ & $1.505(2)$ \\
$\mathrm{O} 5-\mathrm{N} 1$ & $1.2263(18)$ & $\mathrm{C} 10-\mathrm{H} 10$ & 0.9500 \\
$\mathrm{~N} 1-\mathrm{C} 6$ & $1.473(2)$ & $\mathrm{C} 10-\mathrm{C} 11$ & $1.392(2)$ \\
$\mathrm{C} 1-\mathrm{C} 2$ & $1.391(2)$ & $\mathrm{C} 11-\mathrm{C} 12$ & $1.395(2)$ \\
$\mathrm{C} 1-\mathrm{C} 6$ & $1.400(2)$ & $\mathrm{C} 11-\mathrm{C} 14$ & $1.507(2)$ \\
$\mathrm{C} 2-\mathrm{H} 2$ & 0.9500 & $\mathrm{C} 12-\mathrm{H} 12$ & 0.9500 \\
$\mathrm{C} 2-\mathrm{C} 3$ & $1.389(2)$ & $\mathrm{C} 13-\mathrm{H} 13 \mathrm{~A}$ & 0.9800 \\
$\mathrm{C} 3-\mathrm{H} 3$ & 0.9500 & $\mathrm{C} 13-\mathrm{H} 13 \mathrm{~B}$ & 0.9800 \\
$\mathrm{C} 3-\mathrm{C} 4$ & $1.385(3)$ & $\mathrm{C} 13-\mathrm{H} 13 \mathrm{C}$ & 0.9800 \\
$\mathrm{C} 4-\mathrm{H} 4$ & 0.9500 & $\mathrm{C} 14-\mathrm{H} 14 \mathrm{~A}$ & 0.9800 \\
$\mathrm{C} 4-\mathrm{C} 5$ & $1.387(2)$ & $\mathrm{C} 14-\mathrm{H} 14 \mathrm{~B}$ & 0.9800 \\
$\mathrm{C} 5-\mathrm{H} 5$ & 0.9500 & $\mathrm{C} 14-\mathrm{H} 14 \mathrm{C}$ & 0.9800 \\
$\mathrm{C} 5-\mathrm{C} 6$ & $1.385(2)$ & & $123.31(14)$ \\
& & & $117.36(13)$ \\
$\mathrm{O} 1-\mathrm{S} 1-\mathrm{O} 3$ & $109.83(6)$ & $\mathrm{C} 8-\mathrm{C} 7-\mathrm{C} 12$ & 120.8 \\
$\mathrm{O} 1-\mathrm{S} 1-\mathrm{C} 1$ & $106.85(7)$ & $\mathrm{C} 12-\mathrm{C} 7-\mathrm{O} 3$ & $118.42(14)$ \\
$\mathrm{O} 2-\mathrm{S} 1-\mathrm{O} 1$ & $119.41(7)$ & $\mathrm{C} 7-\mathrm{C} 8-\mathrm{H} 8$ & 120.8 \\
$\mathrm{O} 2-\mathrm{S} 1-\mathrm{O} 3$ & $105.16(6)$ & $\mathrm{C} 7-\mathrm{C} 8-\mathrm{C} 9$ & $118.82(14)$ \\
$\mathrm{O} 2-\mathrm{S} 1-\mathrm{C} 1$ & $110.43(7)$ & $\mathrm{C} 9-\mathrm{C} 8-\mathrm{H} 8$ & $\mathrm{C} 8-\mathrm{C} 9-\mathrm{C} 10$ \\
$\mathrm{O} 3-\mathrm{S} 1-\mathrm{C} 1$ & $104.16(6)$ & &
\end{tabular}




\begin{tabular}{|c|c|c|c|}
\hline $\mathrm{C} 7-\mathrm{O} 3-\mathrm{S} 1$ & $120.43(9)$ & $\mathrm{C} 8-\mathrm{C} 9-\mathrm{C} 13$ & $120.43(15)$ \\
\hline $\mathrm{O} 4-\mathrm{N} 1-\mathrm{O} 5$ & $124.23(14)$ & $\mathrm{C} 10-\mathrm{C} 9-\mathrm{C} 13$ & $120.75(15)$ \\
\hline $\mathrm{O} 4-\mathrm{N} 1-\mathrm{C} 6$ & $118.87(12)$ & $\mathrm{C} 9-\mathrm{C} 10-\mathrm{H} 10$ & 118.9 \\
\hline $\mathrm{O} 5-\mathrm{N} 1-\mathrm{C} 6$ & $116.89(13)$ & $\mathrm{C} 11-\mathrm{C} 10-\mathrm{C} 9$ & $122.27(15)$ \\
\hline $\mathrm{C} 2-\mathrm{C} 1-\mathrm{S} 1$ & $115.24(12)$ & $\mathrm{C} 11-\mathrm{C} 10-\mathrm{H} 10$ & 118.9 \\
\hline $\mathrm{C} 2-\mathrm{C} 1-\mathrm{C} 6$ & $118.36(14)$ & $\mathrm{C} 10-\mathrm{C} 11-\mathrm{C} 12$ & $118.57(14)$ \\
\hline $\mathrm{C} 6-\mathrm{C} 1-\mathrm{S} 1$ & $126.38(12)$ & $\mathrm{C} 10-\mathrm{C} 11-\mathrm{C} 14$ & $120.02(14)$ \\
\hline $\mathrm{C} 1-\mathrm{C} 2-\mathrm{H} 2$ & 119.7 & $\mathrm{C} 12-\mathrm{C} 11-\mathrm{C} 14$ & $121.41(14)$ \\
\hline $\mathrm{C} 3-\mathrm{C} 2-\mathrm{C} 1$ & $120.67(15)$ & $\mathrm{C} 7-\mathrm{C} 12-\mathrm{C} 11$ & $118.60(14)$ \\
\hline $\mathrm{C} 3-\mathrm{C} 2-\mathrm{H} 2$ & 119.7 & $\mathrm{C} 7-\mathrm{C} 12-\mathrm{H} 12$ & 120.7 \\
\hline $\mathrm{C} 2-\mathrm{C} 3-\mathrm{H} 3$ & 119.9 & $\mathrm{C} 11-\mathrm{C} 12-\mathrm{H} 12$ & 120.7 \\
\hline $\mathrm{C} 4-\mathrm{C} 3-\mathrm{C} 2$ & $120.11(15)$ & $\mathrm{C} 9-\mathrm{C} 13-\mathrm{H} 13 \mathrm{~A}$ & 109.5 \\
\hline $\mathrm{C} 4-\mathrm{C} 3-\mathrm{H} 3$ & 119.9 & C9-C13-H13B & 109.5 \\
\hline $\mathrm{C} 3-\mathrm{C} 4-\mathrm{H} 4$ & 119.9 & $\mathrm{C} 9-\mathrm{C} 13-\mathrm{H} 13 \mathrm{C}$ & 109.5 \\
\hline $\mathrm{C} 3-\mathrm{C} 4-\mathrm{C} 5$ & $120.10(15)$ & $\mathrm{H} 13 \mathrm{~A}-\mathrm{C} 13-\mathrm{H} 13 \mathrm{~B}$ & 109.5 \\
\hline $\mathrm{C} 5-\mathrm{C} 4-\mathrm{H} 4$ & 119.9 & $\mathrm{H} 13 \mathrm{~A}-\mathrm{C} 13-\mathrm{H} 13 \mathrm{C}$ & 109.5 \\
\hline $\mathrm{C} 4-\mathrm{C} 5-\mathrm{H} 5$ & 120.2 & $\mathrm{H} 13 \mathrm{~B}-\mathrm{C} 13-\mathrm{H} 13 \mathrm{C}$ & 109.5 \\
\hline $\mathrm{C} 6-\mathrm{C} 5-\mathrm{C} 4$ & $119.59(15)$ & $\mathrm{C} 11-\mathrm{C} 14-\mathrm{H} 14 \mathrm{~A}$ & 109.5 \\
\hline $\mathrm{C} 6-\mathrm{C} 5-\mathrm{H} 5$ & 120.2 & $\mathrm{C} 11-\mathrm{C} 14-\mathrm{H} 14 \mathrm{~B}$ & 109.5 \\
\hline $\mathrm{C} 1-\mathrm{C} 6-\mathrm{N} 1$ & $122.82(13)$ & $\mathrm{C} 11-\mathrm{C} 14-\mathrm{H} 14 \mathrm{C}$ & 109.5 \\
\hline $\mathrm{C} 5-\mathrm{C} 6-\mathrm{N} 1$ & $116.05(14)$ & $\mathrm{H} 14 \mathrm{~A}-\mathrm{C} 14-\mathrm{H} 14 \mathrm{~B}$ & 109.5 \\
\hline $\mathrm{C} 5-\mathrm{C} 6-\mathrm{C} 1$ & $121.13(14)$ & $\mathrm{H} 14 \mathrm{~A}-\mathrm{C} 14-\mathrm{H} 14 \mathrm{C}$ & 109.5 \\
\hline $\mathrm{C} 8-\mathrm{C} 7-\mathrm{O} 3$ & $119.06(13)$ & $\mathrm{H} 14 \mathrm{~B}-\mathrm{C} 14-\mathrm{H} 14 \mathrm{C}$ & 109.5 \\
\hline $\mathrm{S} 1-\mathrm{O} 3-\mathrm{C} 7-\mathrm{C} 8$ & $-74.03(15)$ & $\mathrm{C} 1-\mathrm{S} 1-\mathrm{O} 3-\mathrm{C} 7$ & $-84.68(11)$ \\
\hline $\mathrm{S} 1-\mathrm{O} 3-\mathrm{C} 7-\mathrm{C} 12$ & $111.80(13)$ & $\mathrm{C} 1-\mathrm{C} 2-\mathrm{C} 3-\mathrm{C} 4$ & $1.8(2)$ \\
\hline $\mathrm{S} 1-\mathrm{C} 1-\mathrm{C} 2-\mathrm{C} 3$ & $176.26(12)$ & $\mathrm{C} 2-\mathrm{C} 1-\mathrm{C} 6-\mathrm{N} 1$ & $-179.70(14)$ \\
\hline $\mathrm{S} 1-\mathrm{C} 1-\mathrm{C} 6-\mathrm{N} 1$ & $2.0(2)$ & $\mathrm{C} 2-\mathrm{C} 1-\mathrm{C} 6-\mathrm{C} 5$ & $0.8(2)$ \\
\hline $\mathrm{S} 1-\mathrm{C} 1-\mathrm{C} 6-\mathrm{C} 5$ & $-177.44(12)$ & $\mathrm{C} 2-\mathrm{C} 3-\mathrm{C} 4-\mathrm{C} 5$ & $0.0(2)$ \\
\hline $\mathrm{O} 1-\mathrm{S} 1-\mathrm{O} 3-\mathrm{C} 7$ & $29.44(12)$ & $\mathrm{C} 3-\mathrm{C} 4-\mathrm{C} 5-\mathrm{C} 6$ & $-1.3(2)$ \\
\hline $\mathrm{O} 1-\mathrm{S} 1-\mathrm{C} 1-\mathrm{C} 2$ & $21.24(13)$ & $\mathrm{C} 4-\mathrm{C} 5-\mathrm{C} 6-\mathrm{N} 1$ & $-178.57(13)$ \\
\hline $\mathrm{O} 1-\mathrm{S} 1-\mathrm{C} 1-\mathrm{C} 6$ & $-160.46(13)$ & $\mathrm{C} 4-\mathrm{C} 5-\mathrm{C} 6-\mathrm{C} 1$ & $0.9(2)$ \\
\hline $\mathrm{O} 2-\mathrm{S} 1-\mathrm{O} 3-\mathrm{C} 7$ & $159.13(10)$ & $\mathrm{C} 6-\mathrm{C} 1-\mathrm{C} 2-\mathrm{C} 3$ & $-2.2(2)$ \\
\hline $\mathrm{O} 2-\mathrm{S} 1-\mathrm{C} 1-\mathrm{C} 2$ & $-110.09(12)$ & $\mathrm{C} 7-\mathrm{C} 8-\mathrm{C} 9-\mathrm{C} 10$ & $1.6(2)$ \\
\hline $\mathrm{O} 2-\mathrm{S} 1-\mathrm{C} 1-\mathrm{C} 6$ & $68.21(15)$ & $\mathrm{C} 7-\mathrm{C} 8-\mathrm{C} 9-\mathrm{C} 13$ & $-178.43(14)$ \\
\hline $\mathrm{O} 3-\mathrm{S} 1-\mathrm{C} 1-\mathrm{C} 2$ & $137.46(11)$ & $\mathrm{C} 8-\mathrm{C} 7-\mathrm{C} 12-\mathrm{C} 11$ & $0.0(2)$ \\
\hline $\mathrm{O} 3-\mathrm{S} 1-\mathrm{C} 1-\mathrm{C} 6$ & $-44.23(14)$ & $\mathrm{C} 8-\mathrm{C} 9-\mathrm{C} 10-\mathrm{C} 11$ & $-1.1(2)$ \\
\hline $\mathrm{O} 3-\mathrm{C} 7-\mathrm{C} 8-\mathrm{C} 9$ & $-174.87(12)$ & $\mathrm{C} 9-\mathrm{C} 10-\mathrm{C} 11-\mathrm{C} 12$ & $0.0(2)$ \\
\hline $\mathrm{O} 3-\mathrm{C} 7-\mathrm{C} 12-\mathrm{C} 11$ & $173.85(12)$ & $\mathrm{C} 9-\mathrm{C} 10-\mathrm{C} 11-\mathrm{C} 14$ & $-179.82(14)$ \\
\hline $\mathrm{O} 4-\mathrm{N} 1-\mathrm{C} 6-\mathrm{C} 1$ & $-39.7(2)$ & $\mathrm{C} 10-\mathrm{C} 11-\mathrm{C} 12-\mathrm{C} 7$ & $0.6(2)$ \\
\hline $\mathrm{O} 4-\mathrm{N} 1-\mathrm{C} 6-\mathrm{C} 5$ & $139.82(14)$ & $\mathrm{C} 12-\mathrm{C} 7-\mathrm{C} 8-\mathrm{C} 9$ & $-1.1(2)$ \\
\hline $\mathrm{O} 5-\mathrm{N} 1-\mathrm{C} 6-\mathrm{C} 1$ & $141.33(16)$ & $\mathrm{C} 13-\mathrm{C} 9-\mathrm{C} 10-\mathrm{C} 11$ & $178.94(14)$ \\
\hline $\mathrm{O} 5-\mathrm{N} 1-\mathrm{C} 6-\mathrm{C} 5$ & $-39.2(2)$ & $\mathrm{C} 14-\mathrm{C} 11-\mathrm{C} 12-\mathrm{C} 7$ & $-179.61(13)$ \\
\hline
\end{tabular}

Hydrogen-bond geometry $\left(A,{ }^{\circ}\right)$

\begin{tabular}{lllll}
\hline$D-\mathrm{H} \cdots A$ & $D-\mathrm{H}$ & $\mathrm{H} \cdots A$ & $D \cdots A$ & $D-\mathrm{H} \cdots A$ \\
\hline $\mathrm{C} 5-\mathrm{H} 5 \cdots \mathrm{O} 1^{\mathrm{i}}$ & 0.95 & 2.56 & $3.4544(19)$ & 156
\end{tabular}


Symmetry codes: (i) $x+1, y-1, z$; (ii) $x-1, y+1, z$. 